\title{
Comunicação
}

[Communication]

\section{Ocorrência de Siphonaptera em preá Cavia aperea Exerleben, 1777 (Rodentia: Caviidae) no Rio Grande do Sul, Brasil}

\author{
[Occurrence of Siphonaptera in Cavia aperea Exerleben, 1777 (Rodentia: Caviidae) in the state \\ of Rio Grande do Sul, Southern Brazil] \\ C. Krüger, C.S. Mascarenhas, G. Müller, J.G.W. Brum \\ Instituto de Biologia - UFPel \\ 96010-900 - Pelotas, RS
}

Preá, Cavia aperea Exerleben, 1777 (Rodentia: caviidae) ocorre no Brasil, Paraguai, Uruguai e Argentina, e adapta-se com facilidade às mudanças ambientais decorrentes da ação antrópica. C. aperea pode alcançar mais de $25 \mathrm{~cm}$ de comprimento, não possui cauda, e sua pelagem é áspera e de tonalidade grisácea. Vive em bordas de matas, capoeiras, capinzais, gravatazais ou qualquer outro tipo de vegetação baixa e fechada, preferencialmente com água nas proximidades. É abundante no meio rural, podendo, em muitas ocasiões, viver no peridomić́lio e ser utilizada como animal de estimação. Alimenta-se de vegetação herbácea de diferentes espécies e pode invadir plantações de cereais e hortas (Cimardi, 1996; Gonzáles, 2001).

Linardi e Guimarães (2000) citaram em preá, no Brasil, os sifonápteros Adoratopsylla (Tritopsylla) intermedia intermedia (Wagner, 1901), Xenopsylla cheopis (Rothschild, 1903), Polygenis (Neopolygenis) atopus (Jordan \& Rothschild, 1922), Polygenis (Neopolygenis) frustratus (Johnson, 1957), Polygenis (Polygenis) tripus (Jordan, 1933) e Polygenis bohlsi jordani (Lima, 1937). Esta última e mais Ctenocephalides felis felis (Bouché, 1837) também foram citadas em Cavia porcellus (Linnaeus, 1758). Dittmar (2002) examinou 143 espécimes de $C$. aperea no Peru e encontrou os sifonápteros Leptopsylla segnis (Schönherr, 1811) e Tiamastus cavicola (Weyenberg, 1881).

Recebido em 10 de março de 2009

Aceito em 10 de agosto de 2010

E-mail: cristianekruger@gmail.com
O estudo teve por objetivo identificar as espécies de sifonápteros parasitos de $C$. aperea no sul do Rio Grande do Sul, em virtude do pouco conhecimento das espécies que parasitam a preá no estado.

O material constou de 43 preás coletadas na região sul do Rio Grande do Sul, compreendida entre as coordenadas $30^{\circ} 40^{\prime} / 32^{\circ} 18^{\prime} \mathrm{S}$ e $51^{\circ} 23^{\prime} / 53^{\circ} 48^{\prime} \mathrm{W}$, durante maio de 2005 a abril de 2006. Do total, 30 foram capturadas com armadilhas tipo "pit-fall" com intersecção de transecto (Cechin e Martins, 2000) e 13 resultaram de atropelamentos nas rodovias da região. As capturas foram realizadas conforme licença do Instituto Brasileiro do Meio Ambiente e Recursos Naturais Renováveis sob nº 022/2005 - NUFAU.

Os animais capturados nas armadilhas foram anestesiados com éter etílico e transportados para o Laboratório de Parasitologia de Animais Silvestres do Departamento de Microbiologia e Parasitologia, Instituto de Biologia, UFPel, onde foram eutanasiados, de acordo com o Código de Ética da Associação Protetora dos Animais Apa/1989.

Para a coleta dos artrópodes, cada preá foi lavada em um balde contendo água com detergente, e o material resultante tamisado em malha de $150 \mu \mathrm{m}$, sendo os parasitos encontrados acondicionados em frascos com álcool $70^{\circ} \mathrm{GL}$ para posterior sexagem e identificação. Os sifonápteros foram clarificados com salicilato de 
metila, montados entre lâmina e lamínula com bálsamo do Canadá e identificados de acordo com Linardi e Guimarães (2000). Os exemplares foram catalogados e depositados na Coleção de Artrópodes do Laboratório de Parasitologia de Animais Silvestres. Os parâmetros de prevalência, intensidade média e abundância média foram calculados conforme Bush et al. (1997).

Dos 43 animais examinados, 6\% estavam parasitados por Siphonaptera, identificadas como Polygenis (N.) atopus e Polygenis (Polygenis) axius proximus (Guimarães, 1948) (Rhopalopsyllidae).

$P$. (N.) atopus ocorre frequentemente em vários gêneros de roedores e marsupiais, já registrados na Argentina, Bolívia, Brasil, Panamá e Venezuela (Linardi e Guimarães, 2000). No Brasil, há registro nos estados de Minas Gerais, Paraná, Rio de Janeiro, Santa Catarina (Linardi e Guimarães, 2000), São Paulo (Nieri-Basto et al., 2004) e Rio Grande do Sul (Müller et al., 2006).

$P$. (P.) axius proximus foi registrado, no Brasil, apenas em Mato Grosso, Minas Gerais, São
Paulo e Rio Grande do Sul, como parasitos de Akodon arviculoides (Wagner, 1842), Bolomys lasiurus lasiurus (Lund, 1841), Lutreolina crassicaudata (Desmarest, 1804), Rattus rattus (Linnaeus, 1758) (Linardi e Guimarães, 2000) e Didelphis albiventris (Lund, 1841) (Müller et al., 2006).

No Rio Grande do Sul, o primeiro registro de Polygenis (Jordan, 1939), foi realizado por Linardi et al. (2005), que encontraram Polygenis (P.) platensis (Jordan \& Rothschild, 1908) parasitando tuco-tucos (Ctenomys flamarioni Travi, 1981 e Ctenomys minutus, Nehring, 1887) nos municípios de Tramandaí, Capão da Canoa e Osório. Posteriormente, no município de Pelotas, Müller et al. (2006) relataram $P$. (N.) atopus e $P$. $(P$.$) axius proximus em D$. albiventris, com prevalência de $10 \%$ e $3,3 \%$, respectivamente, diferindo de $C$. aperea, que foi de $2,3 \%$ para ambos os sifonápteros.

C. aperea caracteriza um novo hospedeiro para $P$. (N.) atopus e $P$. (P.) axius proximus no Rio Grande do Sul, Brasil.

Palavras-chave: preá, Polygenis, siphonaptera

\begin{abstract}
Due to limited knowledge of Siphonaptera fauna of the Cavia aperea (Brazilian guinea pig) in the State of Rio Grande do Sul, 43 specimens were examined. Six percent of the Brazilian guinea pigs were parasitized with Siphonaptera, which were identified as Polygenis (Neopolygenis) atopus and Polygenis (Polygenis) axius proximus (Rhopalopsyllidae), both with $2.3 \%$ of prevalence. P. (Neopolygenis) atopus and $\mathrm{P}$. (Polygenis) axius proximus are reported for the first time on $\mathrm{C}$. aperea in the State of Rio Grande do Sul, Southern Brazil.
\end{abstract}

Keywords: Brazilian guinea pig, Polygenis, Siphonaptera

\section{REFERÊNCIAS BIBLIOGRÁFICAS}

BUSH, A.O.; LAFFERTY K.D.; LOTZ J.M. et al Parasitology meets ecology on its own terms: Margolis et al. revisited. J. Parasitol., v.83, p.575-583, 1997.

CECHIN, S.Z; MARTINS, M. Eficiência de armadilhas de queda (pitfall traps) em amostragens de anfibios e répteis no Brasil. Rev. Bras. Zool., v.17, p.729-740, 2000.

CIMARDI, A.V. Mamíferos de Santa Catarina. Florianópolis: FATMA, 1996. 302p.
DITTMAR, K. Arthropod and helminth parasites of the guinea pig, Cavia aperea, from the Andes and the Cordillera in Peru, South America. $J$. Parasitol., v.88, p.409-411, 2002.

GONZALES, E.M. Guía de campo de los mamíferos del Uruguay. Introducción al estúdio de los mamíferos. Montevideo: Vida Silvestre, 2001. 339p.

LINARDI, P.M.; GUIMARÃES, L.R. Sifonápteros do Brasil. São Paulo: Museu de Zoologia USP/FAPESP, 2000. 291p. 
LINARDI, P.M.; CARDOSO, V.A.; BOTELHO J.R. et al. Polygenis (Polygenis) platensis (Jordan \& Rothschild) (Siphonaptera: Rhopalopsyllidae, Rhopalopsyllinae), um novo registro no Brasil. Neotrop. Entomol., v.34, p.837-841, 2005.

MÜLLER, G.; LANGONE, P.Q.; MICHELS, G.H. et al. Registro de Sifonápeteros em Didelphis albiventris (gambá-de-orelha-branca) no Rio Grande do Sul, Brasil. In: CONGRESSO BRASILEIRO DE PARASITOLOGIA VETERINÁRIA, 14., 2006, Ribeirão Preto. Anais... São Paulo, 2006. p.242. (Resumo).
NIERI-BASTOS, F.; BARROS-BATTESTI, D.M.; LINARDI, P.M. et al. Ectoparasites of wild rodents from Parque Estadual da Cantareira (Pedra Grande Nuclei), São Paulo, Brazil. Rev. Bras. Parasitol. Vet., v.13, p.29-35, 2004. 